\title{
THE EFFECT OF COLLISIONS ON SPECTRAL LINE FORMATION IN SOLAR MAGNETIC REGIONS
}

\author{
F. K. LA MB \\ Dept. of Theoretical Physics, Oxford University, Oxford, England*
}

\begin{abstract}
The present paper describes the results of a preliminary investigation of the effects on the absorption, emission, and scattering of polarized light caused by collisions between the atoms of interest and surrounding perturbets under physical conditions typical of solar magnetic regions. The description of these effects in terms of atomic level polarization is reviewed and the processes which can lead to atomic level polarization in solar magnetic regions and those which tend to reduce it are summarized. The effects associated with collisional relaxation of atomic level polarization are discussed and a method for estimating relaxation rates is described. Estimates of collisional relaxation rates are used to calculate upper limits on the degree of polarization of the levels involved in the formation of five magnetically-sensitive FeI absorption lines. The implications of the results for the form of radiative transfer equations used to describe the formation of these lines are discussed.
\end{abstract}

\section{Introduction}

The correct interpretation of those solar magnetic field measurements which are based on determination of the polarization of light in magnetically-sensitive solar absorption and emission lines depends on a correct choice of the models of absorption, emission, and scattering processes which are used to describe the formation of these lines. In order to choose models which best describe line formation in solar magnetic regions it is necessary to study the details of the interaction between atoms and polarized light under the physical conditions typical of these regions. Of particular importance for the physical conditions which exist in most solar magnetic regions are the effects on the absorption, emission, and scattering of polarized light due to collisions between the atoms of interest and surrounding perturbers. In spite of intense experimental and theoretical work in recent years (for a review of this work see Berman and Lamb, 1969), the problem of describing these effects remains only partially solved. Nevertheless, this recent work has shown that when collisional relaxation is important many features of the absorption, emission, and scattering of polarized light by atoms may be conveniently described in terms of the density matrices for each of the atomic levels involved in these processes (Cohen-Tannoudji, 1962; Omont, 1965). The atomic level density matrices alone can provide only a partial description of these processes, however, since they cannot give detailed information about the spectral features of the interaction between the atoms of interest and the polarized radiation field. In spite of this limitation, an investigation of the absorption, emission, and scattering of polarized radiation under the physical conditions typical of solar magnetic regions using the level density matrix formalism can still shed much light on the types of effects which are to be expected and can give some criteria for deciding on the importance of collisional relaxation.

\footnotetext{
* Present address: Department of Physics, University of Illinois at Urbana-Champaign, Urbana, Illinois, U.S.A.
} 
The present paper describes some of the results of such a preliminary investigation. The discussion in the following sections will be concerned exclusively with the state of an optically thin atomic assembly exposed to a given radiation environment and the conclusions which it is possible to draw concerning the details of absorption, emission, and scattering of polarized radiation by such an assembly. Although the implications of these conclusions for the form of the radiative transfer equations will be discussed, the problem of solving the radiative transfer equations will not be considered. The features of the interaction of polarized radiation with the atoms of interest which can be treated using the level density matrix formalism may be described by the concept of atomic level polarization: an atomic level will be said to be polarized if the reduced density matrix of the atom within the subspace of the level is not proportional to the unit matrix. In Section 2 we first recall briefly the ways in which atomic level polarization affects the polarization of light in solar absorption and emission lines and then review, in the context of solar magnetic regions, the processes which can lead to atomic level polarization and those which tend to reduce it. The influence of the magnetic field and the role of collisional relaxation are discussed in Section 3, and a way of estimating collisional relaxation rates is described in Section 4. Finally, in Section 5 some of the conclusions which have emerged from this investigation are discussed.

\section{Atomic Level Polarization in Solar Magnetic Regions}

\subsection{The ROLE of atomic level polarization in the polarization of light in a SPECTRAL LINE}

The polarization of light in solar absorption and emission lines may result from the action of one or more of three distinct processes. First, in the presence of a magnetic field the light in absorption or emission lines may become partially polarized simply as the result of Zeeman splitting. Although the radiation absorbed or emitted at each frequency in the line by an assembly of atoms is then at least partially polarized, if atomic level polarization does not accompany the Zeeman splitting and if the assembly is optically thin in the line the total radiation absorbed or emitted, when integrated in frequency over the whole of the line, will be isotropic and unpolarized (one speaks of no 'net' polarization of the line). Second, the light in Zeeman split absorption or emission lines formed in an assembly which is not optically thin may show a different partial polarization at each frequency from that of an optically thin assembly. Even in the absence of atomic level polarization, this phenomenon usually leads to net polarization of the light in the line. Finally, light in absorption or emission lines may become polarized as a result of the polarization of one or both of the atomic levels involved in the formation of the line, since in this case the assembly will preferentially absorb, emit, and scatter radiation of a particular polarization and angular distribution in radiative processes beginning at each of the polarized levels.* Generally

\footnotetext{
* The general phenomenon of level polarization includes the Hanle effect and, more generally, any type of level-crossing interference as special cases.
} 
speaking, atomic level polarization leads to net polarization of the light in the line, whether or not the line also undergoes Zeeman splitting or the assembly is optically thin. It is this last process with which we are concerned in the present paper.

Consider the absorption, emission, and scattering of polarized light in terms of the simplified three-level atomic model shown in Figure 1. In scattering light, the atom undergoes induced absorption from an initial level $A$ (states $a, a^{\prime}, \ldots$ ) to an intermediate (excited) level $B$ (states $\left.b, b^{\prime}, \ldots\right)$ and subsequently makes a transition via spontaneous emission to the final level $C$ (states $c, c^{\prime}, \ldots$ ). The reduced atomic density matrix

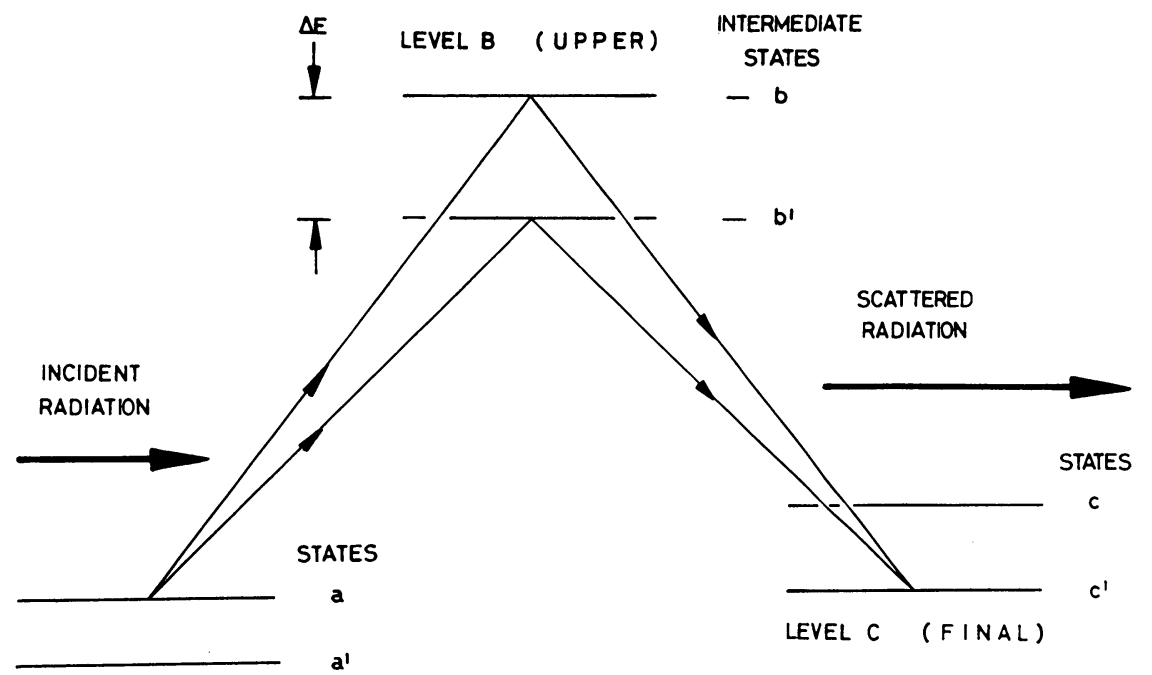

LEVEL A ( INITIAL)

Fig. 1. Simplified three-level atomic model used to illustrate level polarization effects in the absorption, emission, and scattering of polarized light. A typical scattering process is shown in which the atom undergoes induced absorption from an initial level $A$ to an intermediate level $B$ and subsequently makes a transition via spontaneous emission to the final level $C$. In the absence of collisional relaxation, the scattering of a stationary radiation field will produce coherence between the different energy eigenstates of the intermediate level provided the angular frequency separation $\Delta E / \hbar$ between such states is less than or of the order of the radiation damping rate $\Gamma^{R}$ of the same states. If coherence exists between the energy eigenstates of the initial level, this must also be taken into account.

within the subspace of level $A$ will be denoted $\varrho^{A}$, that within $B$ by $\varrho^{B}$, etc. Generally speaking level $C$ may be, but need not be, identical with $A$. However, in the present discussion we wish to focus on the way in which atomic level polarization affects the polarization of light in a particular spectral line involving the two levels $A$ and $B$ and hence the term 'scattering' will be used only to refer to scattering processes of the type $A \rightarrow B \rightarrow A$ in which the initial and final levels (but not necessarily the initial and final states) are identical. Simple absorption or emission in the line is then represented, respectively, by the single transitions $A \rightarrow B$ or $B \rightarrow A$. The polarization of light absorbed in the line will be determined by the polarization of the initial level, that is by $\varrho^{A}$, while the polarization of light emitted into the line will be determined by the 
polarization of the excited level, that is by $\varrho^{B}$. The polarization of light scattered into the line will be determined partially by the polarization of the initial level and partially by the polarization properties of the incident light which takes part in the scattering process; the result may be expressed as a contribution to the polarization of the excited level (and hence to $\varrho^{B}$ ) due to this scattering process.

All present theories of spectral line formation in solar magnetic regions have assumed both that the initial level $A$ is unpolarized and that all processes which populate the excited level $B$ other than direct radiative excitation from level $A$ do not contribute to the polarization of level $B$. In this case the only level polarization which can occur is that of level $B$ in the scattering process $A \rightarrow B \rightarrow A .^{*}$ If level $A$ is populated to some extent by radiative excitation or if level $B$ is populated to some extent by radiative excitation from levels other than $A$, these assumptions must be justified.

\subsection{Processes AfFecting the EXTENT OF LEVEL POlarization IN THE SOlaR ATMOSPHERE}

In an essentially isotropic environment (as, for example, deep in the solar interior) an assembly of atoms will exhibit no level polarization. If, however, the physical environment is not isotropic, the anisotropy will generally lead to atomic level polarization. In the solar atmosphere atomic level polarization might be produced (i) by impact polarization due to collisions with surrounding perturbers which have an anisotropic velocity distribution; (ii) by radiative excitation when the incident radiation is partially polarized or spatially anisotropic; or (iii) by the presence of magnetic fields in conjunction with excitation by radiation, the polarization and intensity of which vary with frequency. In this last case the variation with frequency may itself be produced by Zeeman splitting of atomic levels in the magnetic field. On the other hand atomic level polarization in the solar atmosphere might be reduced (iv) by the presence of a magnetic field; ( $\mathrm{v}$ ) by collisional mixing within the level when the perturber velocity distribution is isotropic; (vi) by collisional mixing with other levels when the perturber velocity distribution is isotropic; or (vii) by radiative decays or recombinations into the level.

Impact polarization is unlikely to be an important source of atomic level polarization, at least in the photosphere and lower chromosphere, since there are strong grounds for believing that the velocity distribution there is essentially isotropic (cf. Böhm, 1960). It may be significant at greater heights in the solar atmosphere, but the present evidence makes this, too, seem unlikely (Charvin, 1965). However, atoms in prominences and the corona will tend to exhibit level polarization because of exposure to anisotropies in the ambient radiation field which are due to the effects of

* Expressions for scattering operators which include this contribution to the polarization of level $B$ have been obtained by Obridko (1965), Rachkovsky (1967), and Domke (1969) for the case when coherence between the different energy eigenstates in level $B$ and collisional relaxation of the level polarization can be neglected. Expressions for scattering operators have also been derived by Stepanov (1962), Rachkovsky (1963), and Domke (1969) for the case when both levels $A$ and $B$ are completely unpolarized. 
limb darkening and the finite angular size of the solar disk. Atoms in the lower chromosphere and photosphere will experience this same radiation anisotropy to a lesser extent. In addition, atoms in solar magnetic regions are subject to level polarization via mechanism (iii). On the other hand, in solar magnetic regions the type of level polarization which can occur will be limited to some extent by the strength of the magnetic field. Throughout the solar atmosphere the polarization of low-lying atomic levels will tend to be reduced by radiative decays into these levels. For atoms located in the lower chromosphere and the photosphere, the polarization of all atomic levels will tend to be reduced by collisional mixing within the level, while level depolarization due to collisional mixing between levels will generally be much less important. The reasons for these conclusions will be described in more detail in the sections which follow.

\section{Collisional Relaxation Effects and the Influence of the Magnetic Field}

As noted in the previous section, the nature and extent of atomic level polarization which occurs in a given solar magnetic region may be profoundly affected both by the magnetic field there and by collisional relaxation. The present section first reviews briefly the way in which level polarization depends on magnetic field strength in the absence of collisions and then discusses the effects of collisional relaxation, including the way in which the dependence on field strength is altered.

\subsection{INFLUENCE OF THE MAGNETIC FIELD}

The strength of the magnetic field in a given solar magnetic region partially determines the type of atomic level polarization which can occur there. The nature of this effect may perhaps be most clearly seen by considering the way in which the forms of the level density matrices depend on the strength of the magnetic field. For the sake of definiteness in the following discussion we shall consider a particular atomic level labelled $S$, but completely analogous considerations hold for other atomic levels.

For atoms without hyperfine structure, ${ }^{*}$ there are two basic regimes which are characterized by the magnitude of the Larmor frequency $\omega_{L}(S)$ for the states in level $S$ relative to the radiation damping rate $\Gamma^{R}(S)$ for these same states. When the strength of the magnetic field is such that $\omega_{L}(S) / \Gamma^{R}(S) \lesssim 1$, coherence between the different energy eigenstates within level $S$ (represented by non-zero off-diagonal elements in the level density matrix $\varrho^{S}$ expressed in the energy representation; cf. Cohen-Tannoud$\mathrm{ji}, 1962)$ is possible and the particular type of level polarization often referred to as level-crossing interference (Franken, 1961) can occur. On the other hand, when the strength of the magnetic field is such that $\omega_{L}(S) / \Gamma^{R}(S) \gg 1$, the level density matrix $\varrho^{S}$ is necessarily diagonal in the energy representation and there can be no coherence between the different energy eigenstates within level $S$. Only in this case can the different types of level polarization which are possible be described in terms of the populations

* Throughout the present paper hyperfine structure is assumed to be absent; the complications which arise when hyperfine structure is present are discussed in Lamb and ter Haar (1971). 
of the energy eigenstates. The precise way in which the field strength $H$ enters expressions for the operators describing absorption, emission, and scattering depends on which of the irreducible tensor components of the atomic level density matrix are involved. For example, expressions for the Stokes parameters $I, Q$, and $U$ depend on both of the two dimensionless parameters $\left(2 \omega_{L} / \Gamma^{R}\right)$ and $\left(\omega_{L} / \Gamma^{R}\right)$, whereas expressions for the Stokes parameter $V$ depend only on $\left(\omega_{L} / \Gamma^{R}\right)$. In order to have a convenient parameter by which to indicate which regime obtains in any given circumstance, we shall refer to the 'critical field strength' $H_{c}$ for which $2 \omega_{L} / \Gamma^{R}=1$. The value of $H_{c}$ depends on the particular atomic level in question and is given by the relation

$$
H_{c}(S)=5.66 \frac{\Gamma^{R}(S)}{g_{J}(S)},
$$

where $H_{c}$ is in gauss, the radiation damping rate $\Gamma^{R}$ is in units of $10^{8} \mathrm{rads}^{-1}$, and $g_{J}$ is the Landé $g$-factor for level $S$.

\subsection{EFFECTS DUE to COLLISIONAL RELAXATION}

In the present subsection the influence on atomic level polarization of collisions between the atoms of interest and surrounding perturbers will be described within the limitations of the level density matrix formalism. Attention will be focussed on the effects of collision-induced transitions between states within the same level while the effects of collision-induced transitions between states in different levels will be largely neglected. The justification for this procedure will be discussed in Section 4.

At very low particle densities where collisional relaxation can be neglected, all $2 j+1$ Zeeman states of a given atomic level with total electronic angular momentum $j$ decay at the same rate, namely the radiation damping rate $\Gamma^{R}$. At higher particle densities the Zeeman states can relax to each other through collisions so that, in general, $(2 j+1)^{2}$ parameters are necessary to describe the decay of the atomic level. However, it has been shown on theoretical grounds that in an isotropic environment the interaction with surrounding perturbers will lead to the independent relaxation of each multipole moment of the atomic density matrix (Fano, 1963; Ben-Reuven, $1966 \mathrm{a}, \mathrm{b})$. For a single atomic level, this leads to a reduction in the number of different level relaxation times, from $(2 j+1)^{2}$ to $2 j+1$, with one relaxation time for each $2^{K}$. pole moment of the level density matrix.

Even when the isotropy of the environment is destroyed by the presence of a magnetic field, it is expected on theoretical grounds that each multipole moment of the density matrix for a single atomic level will relax independently provided the typical frequencies $\omega_{c}$ occurring in the spectrum of the effective collision Hamiltonian (described in Section 4 below) satisfy

$$
\omega_{c} \gg \omega_{L} \text {. }
$$

This result has been confirmed experimentally (see for example Happer and Saloman, 1967; and Omont and Meunier, 1968; the experimental data have been summarized by Berman and Lamb, 1969). For collisions with either electrons or atomic hydrogen at the temperatures typical of solar magnetic regions $\omega_{c} \gtrsim 10^{13} \mathrm{rads}^{-1}$; since $\omega_{L} \lesssim 1.5 \times$ 
$10^{11} \mathrm{rads}^{-1}$ even for the very strongest magnetic fields and largest $\mathrm{g}$-factors of interest, (2) is well-satisfied in these regions.

With the assumption of the independent relaxation of each multipole moment of the density matrix for a single atomic level, the total relaxation rate for a given $2^{K}$-pole moment including both radiation damping and collisional relaxation may be written

$$
\Gamma^{(K)}=\Gamma^{R}+\Gamma_{C}^{(K)},
$$

where $\Gamma_{\mathrm{C}}^{(\mathrm{K})}$ is the collisional relaxation rate of the $K^{\text {th }}$ moment of the atomic level. For absorption, emission, and scattering processes which involve only electric dipole transitions, the only moments of the level density matrices (and hence the only $\left.\Gamma_{\mathrm{C}}^{(\mathbf{K})}\right)$ of any significance are those with $K \leqslant 2$. It may be remarked that while $\Gamma_{\mathrm{C}}^{(1)}$ and $\Gamma_{\mathrm{C}}^{(2)}$, which represent the effects of collision-induced transitions between states within the same atomic level, are comparable to the ordinary collisional broadening rate, $\Gamma_{\mathrm{c}}^{(0)}$ is given by the rate of collision-induced transitions between states in different levels and thus is ordinarily several orders of magnitude smaller at temperatures typical of the solar atmosphere. Both theory and experiment indicate $\Gamma_{\mathrm{C}}^{(1)} \approx \Gamma_{\mathrm{C}}^{(2)}$ (the exact numerical calculation for foreign-gas collisions in the dipole-dipole approximation gives $\Gamma_{\mathrm{C}}^{(1)} / \Gamma_{\mathrm{c}}^{(2)}=1.12 \pm 0.02$; see Berman and Lamb, 1969). Since only very rough estimates of the absolute magnitudes of the $\Gamma_{\mathrm{C}}^{(\mathrm{K})}$ are possible at the present time for the cases of interest to us, no distinction between $\Gamma_{\mathrm{C}}^{(1)}$ and $\Gamma_{\mathrm{C}}^{(2)}$ will be made in the discussion which follows.

Collisional relaxation of atomic level polarization affects the interaction of atoms with the radiation field in two different ways, which may be described intuitively as follows (for a more detailed discussion see Lamb and ter Haar, 1971): (i) the $2^{K}$-pole moments of the atomic level density matrices with $K>0$ relax faster than the total level populations (the $K=0$ moments). This is apparent from the form of Equation (3) and the discussion immediately following it, and results in a reduction of the proportion of polarized radiation associated with absorption, emission, and scattering processes beginning at these levels. If we make the approximations*

$$
\Gamma_{C}^{(1)}=\Gamma_{C}^{(2)} \equiv \Gamma_{C}
$$

and

$$
\Gamma_{c}^{(0)}=0
$$

then the ratio of polarized or anisotropic absorption, emission, or scattering associated with the $K>0$ multipole moments of a given atomic level relative to the isotropic unpolarized absorption, emission, or scattering associated with the $K=0$ moment of the same level is roughly given by

$$
f_{p}=\frac{\Gamma^{R}}{\left(\Gamma^{R}+\Gamma_{c}\right)}
$$

when the level is populated entirely by completely polarized or completely collimated

* Approximation (4) has already been seen to be quite good; as noted earlier, the approximation represented by Equation (5) will be discussed in Section 4. 
radiation in the absence of magnetic fields. If the exciting radiation is not completely collimated or completely polarized, if the level is populated to some extent by isotropic processes (which may include radiative decays, recapture, or collision-induced transitions into the level), or if there is a magnetic field present (see Subsection 3.1), then the importance of polarized absorption, emission, and scattering beginning at the given level will be correspondingly less. (ii) The energy distribution associated with each state in a given atomic level is broadened relative to the energy distribution in the absence of collisions. This makes possible coherence between different energy eigenstates within the same level at higher magnetic field strengths than in the absence of collisions by increasing the energy 'overlap' of these states. Consequently, in the presence of collisional relaxation the critical magnetic field strength $H_{c}$ for a given level, say $S$, is given (within the approximations represented by Equations (4) and (5)) by a modified form of Equation (1), namely

$$
H_{c}(S)=5.66 \frac{\left[\Gamma^{R}(S)+\Gamma_{C}(S)\right]}{g_{J}(S)},
$$

where the radiation damping rate $\Gamma^{R}$ and the multipole collisional relaxation rate $\Gamma_{C}$ are in units of $10^{8} \mathrm{rad} \mathrm{s}^{-1}$. Both effects (i) and (ii) are clearly illustrated by the experimental results (see for example those of Barger, 1967; and Happer and Saloman, 1967, cited earlier).

From these results it seems clear that, except in the corona (see Charvin, 1965), the relative importance of collisional relaxation of atomic level polarization will have a decisive effect on the polarization of spectral lines formed in solar magnetic regions.

\section{Estimating Collisional Relaxation Rates}

\subsection{Qualitative CONCLUSIONS}

In spite of intense work in recent years, the accurate calculation of collisional relaxation rates for the cases of interest here remains beyond the scope of present techniques. Nevertheless, a number of important conclusions emerge from a qualitative analysis of atomic level depolarization by collisions with atomic hydrogen in solar magnetic regions: (i) the impact approximation is valid; (ii) the effects of collision-induced transitions between levels will generally be small; (iii) essentially all collisions are diabatic with respect to the states within the same atomic level (even those collisions with very small impact parameters which are essentially adiabatic with respect to the states within the same atomic level will partially reorient the atom); (iv) collisional relaxation rates are not expected to depend on the strength of the magnetic field for the field strengths and temperatures which exist in solar magnetic regions. The basis for these conclusions is as follows.

The impact approximation is valid (Baranger, 1958) if

$$
n_{H} \pi b_{c}^{3} \ll 1,
$$

where $n_{H}$ is the number density of hydrogen perturbers and $b_{c}$ is the effective collision 
radius. For metal atoms perturbed by collisions with atomic hydrogen at $T \approx 5 \times 10^{3} \mathrm{~K}$, $b_{c}$ is expected to be of order $10^{-7} \mathrm{~cm}$ (a more detailed discussion of this point is given in Lamb and ter Haar, 1971). Since $n_{H} \lesssim 10^{17} \mathrm{~cm}^{-3}$ in the solar atmosphere, the validity condition (8) is certainly satisfied in this case.

Assuming all perturbers are in their ground state and that there are no accidental resonances in the atom-perturber interaction, an effective collision Hamiltonian of second order in the atom-perturber interaction may be introduced by summing over internal perturber variables (cf. Omont and Meunier, 1968; Berman and Lamb, 1969). This effective collision Hamiltonian will have non-vanishing matrix elements only between those atomic states with a separation in angular frequency units $\Delta \omega \lesssim \omega_{c}$, where $\omega_{c}$ is typical of the frequencies occurring in the Fourier transform of the effective collision Hamiltonian. Since the duration of a collision with impact parameter $b$ is $\tau_{d} \approx b / v_{r}$, where $v_{r}$ is the rms relative velocity between atom and perturber, the Fourier transform will contain frequency components up to $\omega_{c}=\tau_{d}^{-1} \approx v_{r} / b_{c}$. For the temperatures and atoms of interest, $b_{c} \approx 10^{-7} \mathrm{~cm}$ and $v_{r} \approx 10^{6} \mathrm{~cm} \mathrm{~s}^{-1}$ giving $\omega_{c} \approx 10^{13}$ $\mathrm{rad} \mathrm{s}^{-1}$. Since for most states lying in different atomic levels $\Delta \omega \gg \omega_{c}$, collision induced transitions between such states will be negligible. This condition may not be completely satisfied in some cases, particularly for the higher levels of the transition metals. For the temperatures of interest, however, we expect only a small contribution to the collisional relaxation rate due to transitions between levels. This is the reason for adopting the approximation $\Gamma_{\mathrm{C}}^{(0)}=0$ in Subsection 3.2.

On the other hand (cf. Spitzer, 1940; Rebane and Rebane, 1966) almost all collisions are diabatic with respect to states within the same atomic level. For Zeeman state separations $\Delta \omega \approx 10^{11} \mathrm{rad} \mathrm{s}^{-1}$, which are the largest occurring in solar magnetic regions, distant collisions at impact parameters out to $b_{\max } \approx 10^{-5} \mathrm{~cm}$ are still diabatic. Only collisions at very large impact parameters $b \gg b_{\max }$ and at very small impact parameters $b \ll b_{c}$ (for which the frequency separation $\delta \omega$ caused by interaction with the perturber is much greater than $\omega_{c}$ ) will be adiabatic for states within the same level. However, even close adiabatic collisions will partially reorient the atom. Because almost all collisions are diabatic in this sense, collisional depolarization rates are likely to be determined largely by the range of the atom-perturber interaction. For the same reason (i.e. because, even for the highest magnetic fields and largest $g$ factors of interest, the Zeeman state separations are much less than $\omega_{c}$ ) collisional depolarization rates are not expected to depend on the strength of the magnetic field in solar magnetic regions.

\subsection{QuANTITATIVE ESTIMATES}

Rough estimates of the rate of collisional relaxation of level polarization have been calculated for a number of atomic levels which are of interest because they are involved in the formation of some of the spectral lines commonly used to study solar magnetic fields. The method of calculation used here is similar to that of Byron and Foley (1964) and Omont (1965), though the approximations used are somewhat more brutal. In addition to the classical path approximation, we have assumed that the 
atom-perturber interaction is adiabatic with respect to states in different atomic levels. It has further been assumed that the atom-perturber interaction is adequately described by the van der Waals term and that this term may be replaced by the London approximation for the static van der Waals interaction between two atoms. Because of these approximations a few words of caution concerning the accuracy of the results are in order. As noted earlier, the assumption of complete adiabaticity with respect to different atomic levels is not satisfied for some of the higher levels of the transition metals. There is also considerable evidence (see for example Behmenburg, 1964; Griem, 1964; Hindmarsh et al., 1967; Roueff and Van Regemorter, 1969) that the atom-perturber interaction is often poorly represented by the van der Waals term. Furthermore, the time dependence of the actual interaction between atom and perturber results in contributions to the effective collision Hamiltonian due to various virtual transitions of the atom and perturber which are different from those given by the London approximation for the static van der Waals interaction (cf. Berman and Lamb, 1969). In spite of these rather serious difficulties, the approximations used here are expected to provide order-of-magnitude estimates which may be useful in identifying cases where collisional level depolarization is either unimportant or essentially complete, but it is clearly not possible at the present time to resolve doubtful cases. On the whole we expect the present method to underestimate the depolarization rate.

\section{Results and Conclusions}

Using atomic level multipole collisional relaxation rates calculated according to the method outlined in Subsection 4.2., estimates of the maximum possible degree of level polarization $f_{p}$ and the critical magnetic field strength $H_{c}$ have been made for the levels involved in a number of magnetically-sensitive solar absorption lines. The results for five $\mathrm{Fe} \mathrm{I}$ lines $(\lambda \lambda 5131,5250,6173,6302$, and 8468$)$ are presented in Table I. The radiative relaxation rates adopted are based on the $f$-values of Corliss and Warner (1964). These represent lower limits since $f$-values for some of the transitions which contribute were unavailable, but it is thought that the most important transitions have been included. The collisional relaxation rates were calculated for an atomic hydrogen density $n_{H}=1.6 \times 10^{17} \mathrm{~cm}^{-3}$ and thermal temperature $T=4500 \mathrm{~K}$, using the estimates of Fe I radii given by Warner (1969). The multipole collisional relaxation rate is expected to depend somewhat more sensitively on atomic hydrogen density than on temperature $\left(\Gamma_{C} \propto n_{H} T^{3 / 10}\right.$ for the van der Waals interaction). When account is also taken of the much greater variation of hydrogen density with height in the solar atmosphere compared to that of the temperature, it is clear that the value of $f_{p}$ for lines formed at heights in the solar atmosphere greater than those corresponding to these values of $n_{H}$ and $T$ will be greater than that given in Table I.

The results presented in Table I indicate that for the atomic levels involved in the formation of the lines listed there, collisional relaxation of atomic level polarization occurs faster than radiative decay at temperatures and neutral hydrogen densities typical of the photosphere. In all cases $f_{p}$ is less than 0.1 and when account is also 
TABLE I

Maximum possible level polarization and critical magnetic field strength for the levels involved in five magnetically-sensitive solar absorption lines

\begin{tabular}{llllll}
\hline$(1)$ & $(2)$ & $(3)$ & $(4)$ & $(5)$ & $(6)$ \\
\hline 5131.478 & $\mathrm{Fe}_{\mathrm{I}}$ & $\ll 1^{\mathrm{a}}$ & $3.4 \times 10^{-3^{\mathrm{c}}}$ & 34 & 43 \\
5250.218 & $\mathrm{Fe}_{\mathrm{I}}$ & $\ll 1^{\mathrm{a}}$ & $4.5 \times 10^{-6^{\mathrm{d}}}$ & $*$ & 31 \\
6173.348 & $\mathrm{Fe}_{\mathrm{I}}$ & $\ll 1^{\mathrm{a}}$ & $8.3 \times 10^{-2^{\mathrm{e}}}$ & 34 & $*$ \\
6302.508 & $\mathrm{Fe}_{\mathrm{I}}$ & $2.1 \times 10^{-2^{\mathrm{b}}}$ & $8.6 \times 10^{-2^{\mathrm{b}}}$ & 44 & $*$ \\
8468.417 & $\mathrm{Fe}_{\mathrm{I}}$ & $\ll 1^{\mathrm{a}}$ & $2.1 \times 10^{-2^{\mathrm{b}}}$ & 34 & 44
\end{tabular}

(1) Wavelength in $\AA$;

(2) Chemical element;

(3) Maximum level polarization $f_{p}$ for the lower level;

(4) Maximum level polarization $f_{p}$ for the upper level;

(5) Critical magnetic field strength $H_{c}$ in gauss for the lower level;

(6) Critical magnetic field strength $H_{c}$ in gauss for the upper level.

a No radiative decays from these levels are listed by Moore (1945); the natural level lifetime is expected to be long.

b The computed radiative relaxation rate $\Gamma^{R}$ includes the transitions $3443,3465,3476,8327,8468$ 8804.

c $\Gamma^{R}$ includes 3397, 4090, 5079, 5131.

d $\Gamma^{R}$ includes 5225,5250 .

e $\Gamma^{R}$ includes $3850,6173,6862$.

i. $\Gamma^{R}$ includes 5230, 5576, 6302 .

* These levels have zero total electronic angular momentum $(j=0)$ and hence cannot be polarized.

taken of the other effects (described in Subsection 2.2) which lead to a reduction in level polarization, the actual degree of level polarization is likely to be an order of magnitude or more smaller than this. In such cases the usual neglect of level polarization in the treatment of absorption and emission processes will be a good approximation and transfer equations which treat scattering assuming complete depolarization may be expected to give a better description of the formation of such lines than those which neglect it. On the other hand, at greater heights in the solar atmosphere collisional relaxation will be less complete. For spectral lines formed primarily at these greater heights, an accurate description of line formation will require a treatment of scattering which includes the effects of partial level depolarization by collisions and an investigation of the level polarization effects described in Subsection 2.1 which appear in absorption and emission.

The results presented in Table $I$ also indicate that in spite of the tendency for collisional relaxation to increase the critical magnetic field strength, $H_{c}$, at photospheric densities the energy 'overlap' of states within the same atomic level will not be significant for the lines listed there when the magnetic field strength is greater than $10^{2} \mathrm{G}$. For this reason and also because of the depolarizing effect of collisions, levelcrossing interference is unlikely to be important for these lines when formed in the lower regions of sunspot magnetic fields. However, level-crossing interference may well 
be important for lines formed in weaker magnetic fields at greater heights in the solar atmosphere.

The present preliminary investigation suggests that, except in the corona, collisional relaxation of atomic level polarization will have a decisive effect on the polarization of spectral lines formed in solar magnetic regions. Much work remains to be done, particularly to improve the reliability of calculated collisional relaxation rates and to explore the spectral features of the interaction between atoms and polarized radiation in the presence of collisions with surrounding perturbers, before an accurate description of the polarization of spectral lines formed in regions where collisional relaxation is only partially complete can be achieved.

\section{References}

Baranger, M.: 1958, Phys. Rev. 111, 481.

Barger, R. L.: 1967, Phys. Rev. 154, 94.

Behmenburg, W.: 1964, J. Quant. Spectr. Radiative Transf. 4, 177.

Ben-Reuven, A.: 1966a, Phys. Rev. 141, 34.

Ben-Reuven, A.: 1966b, Phys. Rev. 145, 7.

Berman, P. R. and Lamb, W. E.: 1969, Phys. Rev. 187, 221.

Böhm, K.-H.: 1960, Stars and Stellar Systems 6, p. 88.

Byron, F. W. and Foley, H. M.: 1964, Phys. Rev. 134, A625.

Charvin, P.: 1965, Ann. Astrophys. 28, 877.

Cohen-Tannoudji, C.: 1962, Ann. Phys. Paris 7, 423.

Corliss, C. H. and Warner, B.: 1964, Astrophys. J. Suppl. 8, 395.

Domke, H.: 1969, Astrofiz. 5, 525.

Fano, U.: 1963, Phys. Rev. 131, 259.

Franken, P. A.: 1961, Phys. Rev. 121, 508.

Griem, H. R.: 1964, Plasma Spectroscopy, McGraw-Hill Publishing Co., Inc., New York.

Happer, W. and Saloman, E. B.: 1967, Phys. Rev. 160, 23.

Hindmarsh, W. R., Petford, A. D., and Smith, G.: 1967, Proc. Roy. Soc. London A297, 296.

Lamb, F. K. and ter Haar, D.: 1971, Phys. Repts., in press.

Moore, C.: 1945, Princeton Obs. Contr., No. 20.

Obridko, V. N.: 1965, Astron. Zh. 42, 102 = Soviet Astron. 9, 77.

Omont, A.: 1965, J. Phys. Paris 26, 26.

Omont, A. and Meunier, J.: 1968, Phys. Rev. 169, 92.

Rachkovsky, D. N.: 1963, Izv. Krymsk. Astrofiz. Obs. 29, 97.

Rachkovsky, D. N.: 1967, Izv. Krymsk. Astrofiz. Obs. 36, 3.

Rebane, V. N. and Rebane, T. K.: 1966, Opt. Spectroskopiya 20, $185=$ Opt. Spectry. 20, 101.

Roueff, E. and Van Regemorter, H.: 1969, Astron. Astrophys. 1, 69.

Spitzer, L.: 1940, Phys. Rev. 58, 348.

Stepanov, V. E.: 1962, Izv. Krymsk. Astrofiz. Obs. 27, 140.

Warner, B.: 1969, Observatory 89, 11.

\section{Discussion}

Pecker: The collisions which enter Lamb's expression for $f_{p}$ are by no means to be confused with the ones which affect the source function. The former concern only those collisions producing a change in the magnetic quantum number.

Lamb: One must distinguish between the different collisional relaxation rates, each describing the relaxation of a different atomic multipole moment, which may affect the form of the source function. The present investigation was primarily concerned with the rate of collisional mixing between states within the same atomic level, which is described by the collisional relaxation rates $\Gamma_{c}^{(K)}(K \neq 0)$ of the 
various higher multipole moments of the individual atomic levels. Collisions with surrounding perturbers will also broaden the absorption and emission line profiles of individual atoms; the broadening of the spectral profile associated with a given multipole transition is described by the collisional relaxation rate of the relevant multipole moment connecting the initial and final levels. Finally, collisions may induce transitions between different atomic levels; the effect of collisions on the total population of a given level may be described by the level monopole collisional relaxation rate $\Gamma_{c}{ }^{(0)}$. In general all of these different relaxation rates will have an important effect on the form of the source function. Although the relaxation rate which enters the expression for the parameter $f_{p}$ describes the relaxation of a different atomic moment than that described by the collisional broadening rate, it is usually of the same order of magnitude.

Pecker: The height dependence of the collisional relaxation rate might in practice bring many difficulties, the 'critical magnetic field' being height-dependent.

Lamb: The critical magnetic field strength $H_{c}$ will vary with height due largely to the dependence of the collisional relaxation rate $\Gamma_{c}$ on the atomic hydrogen density $n_{H}$, and this may complicate the description of line formation if the magnetic field in the line-forming region is of the order of $H_{c}$. 